\title{
A Partitioned PSB Method for Partially Separable Unconstrained Optimization Problems ${ }^{\text {th }}$
}

\author{
Huiping $\mathrm{Cao}^{\mathrm{a}, *}$, Lan $\mathrm{Yao}^{\mathrm{b}}$ \\ ${ }^{a}$ College of Mathematics and Econometrics, Hunan University, Changsha 410082, China \\ ${ }^{b}$ College of Mathematics and Econometrics, Hunan University, Changsha 410082, China
}

\begin{abstract}
In this paper, we propose a partitioned PSB method for solving partially separable unconstrained optimization problems. By using a projection technique, we construct a sufficient descent direction. Under appropriate conditions, we show that the partitioned PSB method with projected direction is globally and superlinearly convergent for uniformly convex problems. In particular, the unit step length is accepted after finitely many iterations. Finally, some numerical results are presented, which show that the partitioned PSB is effective and competitive.
\end{abstract}

Keywords: partially separable optimization problems, partitioned PSB method, projected PSB method, global convergence, superlinear convergence 2010 MSC: 49M15, 65K10, 90C06

\section{Introduction}

We consider the following unconstrained optimization problem

$$
\min f(x), \quad x \in R^{n},
$$

where $f: R^{n} \rightarrow R$ is twice continuously differentiable. We use $g(x), G(x)$ to denote the gradient of $f(x)$ and the Hessian of $f(x)$, respectively.

\footnotetext{
* Corresponding author

Email address: huiping_cao@hnu.edu.cn (Huiping Cao)
}

Preprint submitted to Applied Mathematics and Computation

March 22, 2016

(C) 2016. This manuscript version is made available under the Elsevier user license http://www.elsevier.com/open-access/userlicense/1.0/ 
Quasi-Newton methods are particularly welcome for solving small and medium scale problems due to their superlinear convergence and no necessity to calculate the Hessian of the objective function. A quasi-Newton method typically generates a sequence of iterates $\left\{x_{k}\right\}$ by

$$
x_{k+1}=x_{k}-B_{k}^{-1} g\left(x_{k}\right)
$$

with an approximate Hessian $B_{k}$. The approximation $B_{k}$ usually satisfies the secant condition:

$$
B_{k+1} s_{k}=y_{k}
$$

where $s_{k}=x_{k+1}-x_{k}$ and $y_{k}=g\left(x_{k+1}\right)-g\left(x_{k}\right)$. When $B_{k}$ is positive definite, 5 the quasi-Newton direction $d_{k}=-B_{k}^{-1} g\left(x_{k}\right)$ is a descent direction for $f$ at $x_{k}$. In this case, it is possible to globalize the method by adopting some line search techniques (see e.g., [1, 2, 3, 4]). In this paper, we fucus on the PSB method. If some trust region strategies are adopted, the PSB method has been proved to be globally and superlinearly convergent. But for the line search type PSB method, there is little work about the global convergence, which may result from the non positive definiteness of the approximate Hessian. Recently, An, $\mathrm{Li}$ and Xiao [5] introduced a projection technique and proposed a projected PSB method, which can generate a sufficient descent direction for the objective function. Under certain conditions, the method is globally and superlinearly convergent.

In this paper, we will study the application of the PSB method for solving large-scale problems. A large number of numerical experiments have shown that the approximation $B_{k}$ generated by a quasi-Newton method is generally dense. So the direct use of the quasi-Newton methods is impossible due to the storage difficulty for the matrix $B_{k}$. In order to solve large scale problems, several methods have been proposed. The Limited memory BFGS (L-BFGS) method [6] is an efficient extension of BFGS method for large-scale problems. The method constructs a matrix $H_{k}$, an approximate Hessian of $\nabla^{2} f\left(x_{k}\right)^{-1}$ by updating the basic matrix $H_{0} m$ times using the BFGS formula with previous ${ }_{25} m$ iterations. It has been shown that the L-BFGS method converges linearly. 
Another important kind of sparse quasi-Newton method is that investigating the sparsity of Hessian $\nabla^{2} f(x)$. Suppose that for all $x \in R^{n}$,

$$
\left(\nabla^{2} f(x)\right)_{i, j}=0,(i, j) \in F,
$$

where $F$ is the subset of $I \times I$ and $I=\{1, \ldots, n\}$. Toint [7] and Fletcher [8] have studied the sparse quasi-Newton methods which can satisfies the secant equation and sparse condition simultaneously. However, as pointed out in [9], at each step of the method in [8] or [10, one must solve a convex program or so a linear system. In addition, the approximate Hessian may be ill-posed if some component of $s_{k}$ is zero.

Based on the positive definite matrix completion in semidefinite programming, Yamashita [1] proposed a new sparse quasi-Newton update, called Matrix Completion Quasi-Newton (MCQN) update, for unconstrained optimization problems with sparse Hessian structures. Dai and Yamashita 9] established the local and superlinear convergence for the MCQN update with Broyden's convex family under appropriate assumptions. Besides, the authors gave the related analysis for global convergence assuming that the objective function is uniformly convex and its dimension is only two.

For many large scale problems, the function $f$ has the following partially separable form

$$
\min f(x)=\sum_{i=1}^{m} f_{i}(x),
$$

where each element function $f_{i}, i=1, \ldots, m$ depends only on a few variables. In this case, the partitioned quasi-Newton method [12, 13, performs well in practice. The partitioned BFGS method generates the approximate Hessian $B_{k}^{i}$ for each function $f_{i}$ by the BFGS update. Then quasi-Newton matrix $B_{k}$, as an approximation to the Hessian of $f(x)$ at $x_{k}$, is the summation of $B_{k}^{(i)}, i=1, \ldots, m$. 45 In 1986, Toint [14 established the global convergence of the partitioned BFGS method for solving the partially separable convex problems. Griewank [15] established a stronger global convergence of the partitioned BFGS method. Moreover, it does ensure superlinear convergence with the step sizes going to 1 . 
In this paper, we study the partitioned quasi-Newton method with PSB $1,2, \ldots, m$, function $f_{i}$ is twice continuously differentiable. For each $i=1,2, \ldots$, $m$, we let $g_{i}(x)$ and $G_{i}(x)$ be the gradient and Hessian of $f_{i}$ at $x$. Similar to [14, we suppose that for each $i=1, \ldots, m$, the null space of $G_{i}(x)$, denoted by $\mathcal{N}_{i}$, is independent of $x$. We let $P^{(i)}: R^{n} \rightarrow R^{n}$ be the orthogonal projection $j$-the component can be specified by

$$
x_{j}^{(i)}= \begin{cases}x_{j}, & \text { if } f_{i}(x) \text { depends on } x_{j} \\ 0, & \text { otherwise. }\end{cases}
$$

It is clear that

$$
g(x)=\sum_{i=1}^{m} g_{i}(x)=\sum_{i=1}^{m} g_{i}\left(x^{(i)}\right), G(x)=\sum_{i=1}^{m} G_{i}(x)=\sum_{i=1}^{m} G_{i}\left(x^{(i)}\right) .
$$


Consequently, we have for any $d \in R^{n}$,

$$
G_{i}(x) d=G_{i}\left(x^{(i)}\right) d^{(i)}
$$

where $d^{(i)}=P^{(i)} d$ has the same form as $x^{(i)}$. In a partitioned quasi-Newton method, the approximate Hessian matrix $B_{k}$ takes the form

$$
B_{k}=\sum_{i=1}^{m} B_{k}^{(i)}
$$

with each $B_{k}^{(i)}, i=1, \ldots, m$, an approximation to $G_{i}\left(x_{k}\right)$ that satisfies the secant equation

$$
B_{k+1}^{(i)} s_{k}^{(i)}=y_{k}^{(i)}
$$

where $s_{k}^{(i)}=x_{k+1}^{(i)}-x_{k}^{(i)}$ and $y_{k}^{(i)}=g_{i}\left(x_{k+1}^{(i)}\right)-g_{i}\left(x_{k}^{(i)}\right)$. Matrices $B_{k}^{(i)}, i=$ $1, \ldots, m$ can be updated by any existing update formula. In this paper, we focus on the PSB update. Specifically, for each $i=1, \ldots, m$, we update $B_{k}^{(i)}$ by 75 the following PSB update:

$$
\begin{aligned}
B_{k+1}^{(i)}= & B_{k}^{(i)}+\frac{\left(y_{k}^{(i)}-B_{k}^{(i)} s_{k}^{(i)}\right) s_{k}^{(i) T}+s_{k}^{(i)}\left(y_{k}^{(i)}-B_{k}^{(i)} s_{k}^{(i)}\right)^{T}}{\left\|s_{k}^{(i)}\right\|^{2}} \\
& -\frac{\left(y_{k}^{(i)}-B_{k}^{(i)} s_{k}^{(i)}\right)^{T} s_{k}^{(i)}}{\left\|s_{k}^{(i)}\right\|^{2}} \cdot \frac{s_{k}^{(i)} s_{k}^{(i) T}}{\left\|s_{k}^{(i)}\right\|^{2}}
\end{aligned}
$$

If for some $i=1,2, \ldots, m, s_{k}^{(i)}=0$, we let $B_{k+1}^{(i)}=B_{k}^{(i)}$. These matrices $B_{k}^{(i)}$, $i=1, \ldots, m$, are then accumulated to generate the search direction $d_{k}$ by solving the following system of linear equations

$$
\left(\sum_{i=1}^{m} B_{k}^{(i)}\right) d+g\left(x_{k}\right)=0 .
$$

Then $x_{k+1}$ is determined by

$$
x_{k+1}=x_{k}+\alpha_{k} d_{k},
$$

where $\alpha_{k}$ is a step length that is determined by some line search. When $\alpha_{k} \equiv 1$, we call the above procedure full step partitioned PSB method. The above partitioned PSB method is very similar to the partitioned BFGS method except for the update formula. 
In what follows, we will give several lemmas, which show that the full step partitioned PSB method can retain some good properties of the ordinary PSB method. For simplicity, we denote

$$
A_{k+1}=\sum_{i=1}^{m} A_{k+1}^{(i)} \text {, with } A_{k+1}^{(i)}=\int_{0}^{1} G_{i}\left(x_{k}^{(i)}+\tau s_{k}^{(i)}\right) d \tau, \quad i=1,2, \ldots, m .
$$

It is clear that

$$
A_{k+1}^{(i)} s_{k}^{(i)}=y_{k}^{(i)}, i=1, \ldots, m .
$$

For each $i$ and any $k$, we let $\delta_{k}^{(i)}=0$ if $s_{k}^{(i)}=0$, and

$$
\delta_{k}^{(i)}=\frac{\left\|y_{k}^{(i)}-B_{k}^{(i)} s_{k}^{(i)}\right\|}{\left\|s_{k}^{(i)}\right\|}=\frac{\left\|\left(A_{k+1}^{(i)}-B_{k}^{(i)}\right) s_{k}^{(i)}\right\|}{\left\|s_{k}^{(i)}\right\|} \text {, if } s_{k}^{(i)} \neq 0 .
$$

We also let

$$
\delta_{k}=\frac{\left\|y_{k}-B_{k} s_{k}\right\|}{\left\|s_{k}\right\|}
$$

It is obvious that

$$
\delta_{k} \leq \sum_{i=1}^{m} \delta_{k}^{(i)}
$$

The following lemma is obvious.

Lemma 2.1. Suppose that for each $i=1,2, \ldots, m, G_{i}(x)$ is Lipschitz continuous with a common Lipschitz constant $L>0$, i.e.,

$$
\left\|G_{i}(x)-G_{i}(y)\right\|_{F} \leq L\|x-y\|, \forall x, y, i=1,2, \ldots, m,
$$

where $\|\cdot\|_{F}$ denotes the Frobenius norm of matrices. Then we have

$$
\left\|A_{k+1}^{(i)}-A_{k}^{(i)}\right\|_{F} \leq \frac{1}{2} L\left(\left\|s_{k}^{(i)}\right\|+\left\|s_{k-1}^{(i)}\right\|\right), i=1, \ldots, m .
$$

The following lemma can be proved according to Lemma 3.2 in [5].

Lemma 2.2. Suppose that for each $i=1, \ldots, m, G_{i}(x)$ is Lipschitz continuous with a common Lipschitz constant $L>0$. Let the sequence $\left\{x_{k}\right\}$ be generated by the full step partitioned PSB method. If

$$
\sum_{k=0}^{\infty}\left\|s_{k}\right\|^{2}<\infty
$$


then one has

$$
\lim _{t \rightarrow \infty} \frac{1}{t+1} \sum_{k=0}^{t} \sum_{i=1}^{m} \delta_{k}^{(i)^{2}}=0 .
$$

In particular, there is an infinite index set $K$ independent of $i$ such that the subsequence $\left\{\delta_{k}^{(i)}\right\}_{K}$ converges to zero for any $i=1, \ldots, m$.

If $s_{k}$ satisfies the condition

$$
\sum_{k=0}^{\infty}\left\|s_{k}\right\|<\infty
$$

then one has

$$
\sum_{k=0}^{\infty} \sum_{i=1}^{m} \delta_{k}^{(i)^{2}}<\infty
$$

${ }_{85}$ In particular, for any $i=1, \ldots, m$, the whole sequence $\left\{\delta_{k}^{(i)}\right\}$ converges to zero.

Lemma 2.3. Suppose that for each $i=1, \ldots, m, G_{i}(x)$ is Lipschitz continuous with a common Lipschitz constant $L>0$ and $\sum_{k=0}^{\infty}\left\|s_{k}\right\|^{2}<\infty$. Let the index set $K$ be specified in Lemma 2.2. If there is an accumulation point $x^{*}$ of $\left\{x_{k}\right\}_{K}$ at which $G\left(x^{*}\right)$ is nonsingular, then there is a constant $M_{1}>0$ such that the following inequality holds for all $k \in K$ sufficiently large

$$
\left\|d_{k}\right\| \leq M_{1}\left\|g\left(x_{k}\right)\right\|
$$

Proof. Without loss of generality, we suppose $\left\{x_{k}\right\}_{K} \rightarrow x^{*}$. Since $\sum_{k=0}^{\infty}\left\|s_{k}\right\|^{2}<$ $\infty$, it is clear that when $k \in K$ is sufficiently large, $A_{k+1}$ is nonsingular. Moreover, there is a constant $M_{2}>0$ such that the inequality $\left\|A_{k+1}^{-1}\right\| \leq M_{2}$ holds for all $k \in K$ sufficiently large. It then follows from Lemma 2.2 and 2.2 that 90 $\left\{\delta_{k}\right\}_{K} \rightarrow 0$. Therefore, the inequality $\delta_{k} \leq \frac{1}{2 M_{2}}$ is satisfied for all $k \in K$ sufficiently large. Consequently, we get that for all $k \in K$ sufficiently large,

$$
\begin{aligned}
\left\|d_{k}\right\| & \leq\left\|A_{k+1}^{-1}\right\|\left(\left\|A_{k+1} d_{k}+g\left(x_{k}\right)\right\|+\left\|g\left(x_{k}\right)\right\|\right) \\
& \leq M_{2}\left(\left\|g\left(x_{k}\right)\right\|+\delta_{k}\left\|d_{k}\right\|\right) \\
& \leq M_{2}\left(\left\|g\left(x_{k}\right)\right\|+\frac{1}{2 M_{2}}\left\|d_{k}\right\|\right) \\
& \leq M_{2}\left\|g\left(x_{k}\right)\right\|+\frac{1}{2}\left\|d_{k}\right\| .
\end{aligned}
$$

The last inequality implies 2.6 with $M_{1}=2 M_{2}$. 
The following theorem gives the local and superlinear convergence of the full step partitioned PSB method.

Theorem 2.1. Suppose that for each $i=1, \ldots, m, G_{i}(x)$ is Lipschitz continuous with a common Lipschitz constant $L>0$. Let $x^{*}$ be a solution of the unconstrained optimization problem (1.2) at which $G\left(x^{*}\right)$ is nonsingular. For any $r \in(0,1)$, there exist constants $\epsilon>0$ and $\delta>0$ such that if $\left\|x_{0}-x^{*}\right\| \leq \epsilon$ and $\left\|B_{0}^{(i)}-G_{i}\left(x^{*}\right)\right\| \leq \delta$ for all $i, i=1,2, \ldots, m$, then the sequence generated by the full step partitioned PSB method remains in $U_{\epsilon}\left(x^{*}\right) \triangleq\left\{x \mid\left\|x-x^{*}\right\| \leqslant \epsilon\right\}$. Moreover, the convergence rate of $\left\{x_{k}\right\}$ is superlinear.

\section{A Projected Partitioned PSB Method}

In this section, we will globalize the partitioned PSB method. Like the ordinary PSB method, the matrix $B_{k}$ generated by the partitioned PSB method may not be positive definite. As a result, the generated direction $d_{k}$ may not be a descent direction for $f$ at $x_{k}$. In order to get a descent direction, we adopt a projection technique similar to that in [5]. Suppose $d_{k}^{P S B}$ is the direction generated by the partitioned PSB method. We let $d_{k}$ be defined by

$$
d_{k}=-g\left(x_{k}\right)+\lambda_{k}\left(I-\frac{g\left(x_{k}\right) g\left(x_{k}\right)^{T}}{\left\|g\left(x_{k}\right)\right\|^{2}}\right) d_{k}^{P S B} .
$$

It is a linear combination of the steepest descent direction $-g\left(x_{k}\right)$ and the projection of $d_{k}^{P S B}$ onto the subspace orthogonal to $-g\left(x_{k}\right)$. It follows from (3.1) that

$$
g\left(x_{k}\right)^{T} d_{k}=-\left\|g\left(x_{k}\right)\right\|^{2} .
$$

Consequently, $d_{k}$ is a sufficiently descent direction for function $f$ at $x_{k}$. In the case where $g\left(x_{k}\right)^{T} d_{k}^{P S B}<0$, we can take $\lambda_{k}=-\frac{\left\|g\left(x_{k}\right)\right\|^{2}}{g\left(x_{k}\right)^{T} d_{k}^{P S B}}$ so that we get a descent direction parallel to $d_{k}^{P S B}$, i.e., it holds that

$$
d_{k}=\lambda_{k} d_{k}^{P S B}
$$

As we have had a descent direction, we can determine the step length $\alpha_{k}$ by letting it satisfy the Armijo condition or the Wolfe conditions. The Armijo 
line search is find a step length $\alpha_{k}=\max \left\{\beta_{k} \rho^{i}, i=0,1, \ldots\right\}$ such the following inequality

$$
f\left(x_{k}+\alpha_{k} d_{k}\right) \leq f\left(x_{k}\right)+\sigma_{1} \alpha_{k} g\left(x_{k}\right)^{T} d_{k}, k=1,2, \ldots
$$

holds, where $\rho \in(0,1), \beta_{k} \geq \beta^{*}, \beta^{*}>0$ and $\sigma_{1} \in(0,1)$. The Wolfe conditions is find a step length $\alpha_{k}$ satisfying

$$
\left\{\begin{array}{l}
f\left(x_{k}+\alpha_{k} d_{k}\right) \leq f\left(x_{k}\right)+\sigma_{1} \alpha_{k} g\left(x_{k}\right)^{T} d_{k}, \\
g\left(x_{k}+\alpha_{k} d_{k}\right)^{T} d_{k} \geq \sigma_{2} g\left(x_{k}\right)^{T} d_{k},
\end{array}\right.
$$

where $0<\sigma_{1}<\sigma_{2}<1, \sigma_{1}<1 / 2$.

Based on the above arguments, we propose a damped version of the partitioned PSB method as follows.

Algorithm 3.1. Projected Partitioned PSB Method (P-PSB)

Step 0. Given constants $\lambda>0$ and $\varepsilon>0$. Chosen initial point $x_{0} \in R^{n}$ and initial symmetric matrices $B_{0}^{(i)}, i=1,2, \ldots, m$. Set

$$
B_{0}=\sum_{i=1}^{m} B_{0}^{(i)} .
$$

Let $k:=0$.

Step 1. Stop, if $\left\|g\left(x_{k}\right)\right\| \leq \varepsilon$. Otherwise, solve the system of linear equations 2.1 to get $d_{k}^{P S B}$.

Step 2. Compute $d_{k}$ by (3.1), where $\lambda_{k} \in R^{n}$ is determined by

$$
\lambda_{k}= \begin{cases}0, & \text { if } g\left(x_{k}\right)^{T} d_{k}^{P S B}=0, \\ \min \left\{\lambda k^{1 / 4}, \frac{\left\|g\left(x_{k}\right)\right\|^{2}}{\left|g\left(x_{k}\right)^{T} d_{k}^{P S B}\right|}\right\}, & \text { otherwise }\end{cases}
$$

Step 3. Let $\alpha_{k}$ be determined by the Armijo line search 3.2 or Wolfe line search 3.3. Let $x_{k+1}:=x_{k}+\alpha_{k} d_{k}$.

Step 4. If for some $i=1,2, \ldots, m, \bar{s}_{k}^{(i)}=0$, we let $B_{k+1}^{(i)}=B_{k}^{(i)}$. Other- 
wise, update $B_{k}^{(i)}$ by the PSB update formula

$$
\begin{aligned}
B_{k+1}^{(i)}= & B_{k}^{(i)}+\frac{\left(\bar{y}_{k}^{(i)}-B_{k}^{(i)} \bar{s}_{k}^{(i)}\right) \bar{s}_{k}^{(i) T}+\bar{s}_{k}^{(i)}\left(\bar{y}_{k}^{(i)}-B_{k}^{(i)} \bar{s}_{k}^{(i)}\right)^{T}}{\left\|\bar{s}_{k}^{(i)}\right\|^{2}} \\
& -\frac{\left(\bar{y}_{k}^{(i)}-B_{k}^{(i)} \bar{s}_{k}^{(i)}\right)^{T} \bar{s}_{k}^{(i)}}{\left\|\bar{s}_{k}^{(i)}\right\|^{2}} \cdot \frac{\bar{s}_{k}^{(i)} \bar{s}_{k}^{(i) T}}{\left\|\bar{s}_{k}^{(i)}\right\|^{2}}
\end{aligned}
$$

where $\bar{s}_{k}^{(i)}=\bar{\alpha}_{k} d_{k}^{P S B(i)}, \bar{\alpha}_{k}=\lambda_{k} \alpha_{k}$ and $\bar{y}_{k}^{(i)}=g_{i}\left(x_{k}^{(i)}+\bar{s}_{k}^{(i)}\right)-g_{i}\left(x_{k}^{(i)}\right)$. Let $B_{k+1}$ be determined by

$$
B_{k+1}=\sum_{i=0}^{m} B_{k+1}^{(i)}
$$

Step 5. Let $k:=k+1$. Go to step 1 .

In the Armijo line search, $\beta_{k}$ is determined by

$$
\beta_{k}= \begin{cases}1, & \text { if } g\left(x_{k}\right)^{T} d_{k}^{P S B} \geq 0, \\ \max \left\{\beta^{*}\left\|g\left(x_{k}\right)\right\|^{\tau}, \frac{\left\|g\left(x_{k}\right)\right\|^{2}}{\left|g\left(x_{k}\right)^{T} d_{k}^{P S B}\right|}\right\}, & \text { otherwise }\end{cases}
$$

where $\beta^{*}>0$ and $\tau>0$ are constants.

Remark. Matrix $B_{k+1}$ determined by (3.4) and (3.5) may be singular even if $B_{k}$ is nonsingular. To overcome this drawback, we can adopt the same ideas in [5, 16] to give some analysis of nonsingular partitioned PSB update.

In what follows, for tridiagonal partially separable function, i.e., the Hessian of function $f$ is tridiagonal matrix, one has $m=n-1$. In this case, we modify the partitioned PSB update as follows. We rewrite $B_{k+1}$ as

$$
\begin{aligned}
B_{k+1}= & \sum_{i=1}^{n-1} B_{k+1}^{(i)}=\sum_{i=1}^{n-1}\left(B_{k}^{(i)}+\frac{\left(\bar{y}_{k}^{(i)}-B_{k}^{(i)} \bar{s}_{k}^{(i)}\right) \bar{s}_{k}^{(i) T}+\bar{s}_{k}^{(i)}\left(\bar{y}_{k}^{(i)}-B_{k}^{(i)} \bar{s}_{k}^{(i)}\right)^{T}}{\left\|\bar{s}_{k}^{(i)}\right\|^{2}}\right. \\
& \left.-\frac{\left(\bar{y}_{k}^{(i)}-B_{k}^{(i)} \bar{s}_{k}^{(i)}\right)^{T} \bar{s}_{k}^{(i)}}{\left\|\bar{s}_{k}^{(i)}\right\|^{2}} \cdot \frac{\bar{s}_{k}^{(i)}\left(\bar{s}_{k}^{(i)}\right)^{T}}{\left\|\bar{s}_{k}^{(i)}\right\|^{2}}\right) \\
= & B_{k}+\sum_{i=1}^{n-1}\left(\frac{\left(\bar{y}_{k}^{(i)}-B_{k}^{(i)} \bar{s}_{k}^{(i)}\right) \bar{s}_{k}^{(i) T}+\bar{s}_{k}^{(i)}\left(\bar{y}_{k}^{(i)}-B_{k}^{(i)} \bar{s}_{k}^{(i)}\right)^{T}}{\left\|\bar{s}_{k}^{(i)}\right\|^{2}}\right. \\
& \left.-\frac{\left(\bar{y}_{k}^{(i)}-B_{k}^{(i)} \bar{s}_{k}^{(i)}\right)^{T} \bar{s}_{k}^{(i)}}{\left\|\bar{s}_{k}^{(i)}\right\|^{2}} \cdot \frac{\bar{s}_{k}^{(i)}\left(\bar{s}_{k}^{(i)}\right)^{T}}{\left\|\bar{s}_{k}^{(i)}\right\|^{2}}\right)
\end{aligned}
$$


where for $i=1$, we let

$$
B_{k}^{(1)}=\left(\begin{array}{ccccc}
B_{k}(1,1) & B_{k}(1,2) & 0 & \cdots & 0 \\
B_{k}(2,1) & 0 & 0 & \cdots & 0 \\
0 & 0 & 0 & \cdots & 0 \\
\vdots & \vdots & \vdots & \ddots & \vdots \\
0 & 0 & 0 & \cdots & 0
\end{array}\right)
$$

and for $i=2, \ldots, n-1$, we let

$$
B_{k}^{(i)}=\left(\begin{array}{cccccc}
0 & \cdots & 0 & 0 & \cdots & 0 \\
\vdots & \ddots & \vdots & \vdots & \ddots & \vdots \\
0 & \cdots & B_{k}(i, i) & B_{k}(i, i+1) & \cdots & 0 \\
0 & \cdots & B_{k}(i+1, i) & B_{k}(i+1, i+1) & \cdots & 0 \\
\vdots & \ddots & \vdots & \vdots & \ddots & \vdots \\
0 & \cdots & 0 & 0 & \cdots & 0
\end{array}\right) .
$$

Set $C_{0}=B_{k}$ and define for $i=1,2, \ldots, n-1$

$$
\begin{aligned}
C_{i}= & C_{0}+\sum_{j=1}^{i} \theta_{j} \frac{\left(\bar{y}_{k}^{(i)}-B_{k}^{(i)} \bar{s}_{k}^{(i)}\right) \bar{s}_{k}^{(i) T}+\bar{s}_{k}^{(i)}\left(\bar{y}_{k}^{(i)}-B_{k}^{(i)} \bar{s}_{k}^{(i)}\right)^{T}}{\left\|\bar{s}_{k}^{(i)}\right\|^{2}} \\
& \left.-\theta_{j}^{2} \frac{\left(\bar{y}_{k}^{(i)}-B_{k}^{(i)} \bar{s}_{k}^{(i)}\right)^{T} \bar{s}_{k}^{(i)}}{\left\|\bar{s}_{k}^{(i)}\right\|^{2}} \cdot \frac{\bar{s}_{k}^{(i)}\left(\bar{s}_{k}^{(i)}\right)^{T}}{\left\|\bar{s}_{k}^{(i)}\right\|^{2}}\right) \\
= & C_{i-1}+\theta_{i} \frac{\left(\bar{y}_{k}^{(i)}-B_{k}^{(i)} \bar{s}_{k}^{(i)}\right) \bar{s}_{k}^{(i) T}+\bar{s}_{k}^{(i)}\left(\bar{y}_{k}^{(i)}-B_{k}^{(i)} \bar{s}_{k}^{(i)}\right)^{T}}{\left\|\bar{s}_{k}^{(i)}\right\|^{2}} \\
& \left.-\theta_{i}^{2} \frac{\left(\bar{y}_{k}^{(i)}-B_{k}^{(i)} \bar{s}_{k}^{(i)}\right)^{T} \bar{s}_{k}^{(i)}}{\left\|\bar{s}_{k}^{(i)}\right\|^{2}} \cdot \frac{\bar{s}_{k}^{(i)}\left(\bar{s}_{k}^{(i)}\right)^{T}}{\left\|\bar{s}_{k}^{(i)}\right\|^{2}}\right)
\end{aligned}
$$

${ }_{125}$ Note that $C_{0}^{(i)}=C_{1}^{(i)}=\cdots=C_{i-1}^{(i)}$, then

$$
\begin{aligned}
C_{i}= & C_{i-1}+\theta_{i} \frac{\left(\bar{y}_{k}^{(i)}-C_{i-1}^{(i)} \bar{s}_{k}^{(i)}\right) \bar{s}_{k}^{(i) T}+\bar{s}_{k}^{(i)}\left(\bar{y}_{k}^{(i)}-C_{i-1}^{(i)} \bar{s}_{k}^{(i)}\right)^{T}}{\left\|\bar{s}_{k}^{(i)}\right\|^{2}} \\
& \left.-\theta_{i}^{2} \frac{\left(\bar{y}_{k}^{(i)}-C_{i-1}^{(i)} \bar{s}_{k}^{(i)}\right)^{T} \bar{s}_{k}^{(i)}}{\left\|\bar{s}_{k}^{(i)}\right\|^{2}} \cdot \frac{\bar{s}_{k}^{(i)}\left(\bar{s}_{k}^{(i)}\right)^{T}}{\left\|\bar{s}_{k}^{(i)}\right\|^{2}}\right)
\end{aligned}
$$

For a scalar $\alpha \in(0,1), \theta_{i}$ can be chosen such that

$$
\left|\operatorname{det} C_{i}\right| \geq|\sqrt[n]{\alpha}| \operatorname{det} C_{i-1} \mid, \theta_{i} \in\left[\frac{1-\sqrt[n]{\alpha}}{1+\sqrt[n]{\alpha}}, 1\right] .
$$


therefore we an get $\left|\operatorname{det}\left(B_{k+1}\right)\right| \geq \alpha\left|\operatorname{det} B_{k}\right|$, and $\theta_{i}$ can be chosen so that

$$
B_{k+1} \text { is nonsigular, and }\left|\theta_{i}-1\right| \leq \hat{\theta}<1 \text {. }
$$

The dependence of $\theta_{i}$ on the iteration $k$ is suppressed, but note that $\hat{\theta}$ is independent of $k$ [17.

To prove the global convergence of Algorithm 3.1, we need the following assumptions.

Assumption 3.1. (a) Function $f(x)$ is twice continuously differentiable in the level set $\Omega=\left\{x \mid f(x) \leq f\left(x_{0}\right)\right\}$, and for each $i=1,2, \ldots, m, G_{i}(x)$ is Lipschitz continuous with a common constant $L>0$ such that

$$
\left\|G_{i}(x)-G_{i}(y)\right\|_{F} \leq L\|x-y\|, \forall x, y \in \Omega
$$

(b) Function $f(x)$ is uniformly convex, that is, there exist constants $M \geq$ $m>0$ such that

$$
m\|p\|^{2} \leq p^{T} G(x) p \leq M\|p\|^{2}, \forall p \in R^{n}, \forall x \in \Omega .
$$

Let $x^{*}$ be the unique solution of problem (1.2). It can be obtained according to the conditions of Assumption 3.1 that there are constants $M \geq m>0$ such that [2]

$$
\frac{1}{2} m\left\|x-x^{*}\right\|^{2} \leq f(x)-f\left(x^{*}\right) \leq \frac{1}{2} M\left\|x-x^{*}\right\|^{2}, \forall x \in \Omega
$$

and

$$
\|g(x)\| \geq m\left\|x-x^{*}\right\|, \forall x \in \Omega .
$$

Firstly, similar to Lemma 3.1 in [5], we have the following lemma.

Lemma 3.1. Let the conditions in Assumption 3.1 hold and the sequence $\left\{x_{k}\right\}$ be generated by Algorithm 3.1. Then one has

$$
\sum_{k=0}^{\infty}\left\|x_{k+1}-x_{k}\right\|^{2}=\sum_{k=0}^{\infty}\left\|s_{k}\right\|^{2}<\infty
$$

which implies

$$
\sum_{k=0}^{\infty}\left\|\bar{s}_{k}\right\|^{2}<\infty
$$


For the sake of convenience, for each $i$ and any $k$, we let $\xi_{k}^{(i)}=0$ if $\bar{s}_{k}^{(i)}=0$,

$$
\xi_{k}^{(i)}=\frac{\left\|\bar{y}_{k}^{(i)}-B_{k}^{(i)} \bar{s}_{k}^{(i)}\right\|}{\left\|\bar{s}_{k}^{(i)}\right\|}=\frac{\left\|\left(\bar{A}_{k+1}^{(i)}-B_{k}^{(i)}\right) d_{k}^{P S B^{(i)}}\right\|}{\left\|d_{k}^{P S B^{(i)}}\right\|} \text {, if } \bar{s}_{k}^{(i)} \neq 0 .
$$

and

$$
\xi_{k}=\frac{\left\|\bar{y}_{k}-B_{k} \bar{s}_{k}\right\|}{\left\|\bar{s}_{k}\right\|}=\frac{\left\|\left(\bar{A}_{k+1}-B_{k}\right) \bar{s}_{k}\right\|}{\left\|\bar{s}_{k}\right\|}=\frac{\left\|\left(\bar{A}_{k+1}-B_{k}\right) d_{k}^{P S B}\right\|}{\left\|d_{k}^{P S B}\right\|},
$$

where $\bar{A}_{k+1}=\int_{0}^{1} G\left(x_{k}+t \bar{s}_{k}\right)$. By the definitions of $\xi_{k}$ and $\xi_{k}^{(i)}, i=1, \ldots, m$, we can derive

$$
\xi_{k} \leq \sum_{i=1}^{m} \xi_{k}^{(i)}
$$

The following result can be proved in a way similar to Lemma 3.3 in [5].

Lemma 3.2. Let the conditions in Assumption 3.1 hold and the sequence $\left\{x_{k}\right\}$ be generated by Algorithm 3.1. Then one has

$$
\lim _{t \rightarrow \infty} \frac{1}{t+1} \sum_{k=0}^{t} \sum_{i=1}^{m} \xi_{k}^{(i)^{2}}=0 .
$$

In particular, there is an infinite index set $K$ independent of $i$ such that the subsequence of $\left\{\xi_{k}^{(i)}\right\}_{K}$ converges to zero for any $i=1, \ldots, m$.

If $\left\{s_{k}\right\}$ satisfies the condition

$$
\sum_{k=0}^{\infty}\left\|\bar{s}_{k}\right\|<\infty
$$

then one has

$$
\sum_{k=0}^{\infty} \sum_{i=1}^{m} \xi_{k}^{(i)^{2}}<\infty
$$

In particular, for any $i=1, \ldots, m$, the whole sequence $\left\{\xi_{k}^{(i)}\right\}$ converges to zero.

The following theorem establishes the global convergence of Algorithm 3.1.

Theorem 3.1. Let the conditions in Assumption 3.1 hold and the sequence $\left\{x_{k}\right\}$ be generated by Algorithm 3.1. Then $\left\{x_{k}\right\}$ converges to the unique solution of (1.2) in $\Omega$. 
Proof. According to Lemma 3.2 , there is a subsequence $\left\{\xi_{k}\right\}_{k \in K}$ of $\left\{\xi_{k}\right\}$ defined by (3.11 converges to zero. Since $\left\{x_{k}\right\}_{k \in K} \subseteq \Omega$ is bounded, without loss of generality, we assume that $\left\{x_{k}\right\}_{k \in K}$ itself converges to some point $\bar{x}$. Because $\left\|\overline{s_{k}}\right\| \rightarrow 0$ as $k \rightarrow \infty$, so $\left\{\bar{A}_{k+1}\right\}_{k \in K} \rightarrow G(\bar{x})$. In particular, there exists a constant $\bar{M}_{2}>0$ such that $\left\|\bar{A}_{k+1}^{-1}\right\| \leq \bar{M}_{2}$ for all $k \in K$ sufficiently large. Thus we have

$$
\begin{aligned}
\left\|d_{k}^{P S B}\right\| & \leq\left\|\bar{A}_{k+1}^{-1}\left(\left(\bar{A}_{k+1}-B_{k}\right) d_{k}^{P S B}-g\left(x_{k}\right)\right)\right\| \\
& \leq\left\|\bar{A}_{k+1}^{-1}\right\|\left(\left\|\left(\bar{A}_{k+1}-B_{k}\right) d_{k}^{P S B}\right\|+\left\|g\left(x_{k}\right)\right\|\right) \\
& \leq \bar{M}_{2}\left(\xi_{k}\left\|d_{k}^{P S B}\right\|+\left\|g\left(x_{k}\right)\right\|\right) .
\end{aligned}
$$

Since $\left\{\xi_{k}\right\}_{k \in K} \rightarrow 0$ as $k \rightarrow \infty$ with $k \in K$, the last inequality implies that there is a constant $\bar{M}_{1}>0$ such that the following inequality

$$
\left\|d_{k}^{P S B}\right\| \leq \bar{M}_{1}\left\|g\left(x_{k}\right)\right\|
$$

is satisfied for all $k \in K$ sufficiently large.

By the definition of $d_{k}$, we have

$$
\begin{aligned}
\left\|d_{k}+g\left(x_{k}\right)\right\|^{2} & =\lambda_{k}^{2}\left\|\left(I-\frac{g\left(x_{k}\right) g\left(x_{k}\right)^{T}}{\left\|g\left(x_{k}\right)\right\|^{2}}\right) d_{k}^{P S B}\right\|^{2} \\
& \leq \lambda_{k}^{2}\left\|d_{k}^{P S B}\right\|^{2} \\
& \leq \lambda^{2} \bar{M}_{1}^{2} k^{1 / 2}\left\|g\left(x_{k}\right)\right\|^{2}
\end{aligned}
$$

where the last inequality follows from the definition of $\lambda_{k}$ and (3.15).

If $\liminf _{k \rightarrow \infty}\left\|g\left(x_{k}\right)\right\| \neq 0$, then there is a constant $\eta>0$ such that $\left\|g\left(x_{k}\right)\right\| \geq$ $\eta$ for all $k$. Consequently, the inequality 3.16 yields

$$
\left\|d_{k}+g\left(x_{k}\right)\right\|^{-2} \geq \lambda^{-2} \bar{M}_{1}^{-2} \eta^{-2} k^{-1 / 2},
$$

which implies

$$
\sum_{k=1}^{\infty}\left\|d_{k}+g\left(x_{k}\right)\right\|^{-2}=+\infty .
$$

This yields a contradiction with Theorem 2.2 in [5]. Consequently, it must hold that

$$
\liminf \left\|g\left(x_{k}\right)\right\|=0
$$


Moreover, the step length $\alpha_{k}=\beta_{k}$ is accepted.

Lemma 3.4. Let the conditions in Assumption 3.1 hold and the sequence $\left\{x_{k}\right\}$ be generated by Algorithm 3.1 with $\alpha_{k}$ determined by Armijo line search (3.2) with $0<\sigma_{1}<1 / 2$. Then we have

$$
\sum_{k=0}^{\infty}\left\|x_{k}-x^{*}\right\|<\infty
$$

As a direct result of (3.17), we have

$$
\sum_{k=0}^{\infty}\left\|\bar{s}_{k}\right\| \leq \sum_{k=0}^{\infty}\left\|s_{k}\right\|=\sum_{k=0}^{\infty}\left\|x_{k+1}-x_{k}\right\| \leq \sum_{k=0}^{\infty}\left(\left\|x_{k+1}-x^{*}\right\|+\left\|x_{k}-x^{*}\right\|\right)<\infty .
$$

Therefore, we get from Lemma 3.2 that

$$
\sum_{k=0}^{\infty} \sum_{i=1}^{m} \xi_{k}^{(i)^{2}}<\infty
$$

In particular, for any $i=1,2, \ldots$, the whole sequence $\left\{\xi_{k}^{(i)}\right\}$ converges to zero.

In what follows, we give the superlinear convergence of Algorithm 3.1.

Theorem 3.2. Let the conditions in Assumption 3.1 hold and the sequence

$\left\{x_{k}\right\}$ be generated by Algorithm 3.1 with $\alpha_{k}$ determined by Armijo line search (3.2) with $0<\sigma_{1}<1 / 2$. Then $\left\{x_{k}\right\}$ converges to $x^{*}$ superlinearly. 


\section{Numerical Experiments}

In this section, we test the performance of the proposed partitioned PSB (P-PSB) method on 30 problems [18, 19, 20, where the Hessians of the tested problems have various sparse structures. We also compare the P-PSB method with the projected PSB (PSB) method [5], the partitioned BFGS (P-BFGS) method and limited memory BFGS (L-BFGS) method. The numerical experiments are done by using MATLAB v7.10 on a Core (TM) 2 PC. The parameters in Algorithm 3.1 are specified as follows:

$$
\lambda=10^{3}, \beta^{*}=10^{-7}, \tau=\frac{1}{8}, \sigma_{1}=10^{-4}, \sigma_{2}=0.9 .
$$

For the L-BFGS method, $m=12$, which is the number of stored vectors of L-BFGS, is set, and the scaling factor $s_{k-1}^{T} y_{k-1} /\left\|y_{k-1}\right\|^{2}$ is employed.

In the experiments, we employ the following termination criterion:

$$
\left\|\nabla f\left(x_{k}\right)\right\|_{2}<10^{-5} \cdot \max \left(1,\left\|x_{k}\right\|_{2}\right) \text { or } k \geq 5000
$$

where the second criterion implies that the method fails to obtain a solution.

To show the advantage of the P-PSB method in computation, we test the P-PSB method, the P-BFGS method and the L-BFGS method on 30 partially separable problems with different dimensions, where the size varies from 10 to $2 \times 10^{6}$. Noticing that the number of iterations is virtually independent of the problem size, so we just list a small set of sizes to illustrate the behaviour of the three methods. We give the numerical results in Tables 1, 2 and 3, where the meaning of each column is listed below.

Pro: the number of the test problem;

$\mathrm{n}$ : the dimension of the problem;

Ite: the total number of iterations;

fcnt: the total number of function evaluations;

grad: the total number of gradient evaluations; 
time: the total CPU time (sec).

Results presented in Tables 1, 2 and 3 advocate the proposed P-PSB method. on 7 problems $(8,11,15,19,20,21,29)$. It seems that the advantage on CPU time for the proposed method is not obvious.

In order to analyse the efficiency of proposed method more precisely, we adopt a notion of performance profile [21, which is a distribution function for a performance metric to evaluate and compare the performance of the set of solvers $S$ on a test set $P$. Suppose that there exist $N_{s}$ solvers and $N_{p}$ problems, for each problem $p$ and solver $s$, Dolan and Moré [21] defined $t_{p, s}$ the number of iterations (the number of function evaluations or others) required to solve problem $p$ by solver $s$. Requiring a baseline for comparisons, they compared the performance on problem $p$ by solver $s$ with the best performance by any solver on this problem; i.e., using the performance ratio

$$
r_{p, s}=\frac{t p, s}{\min \left\{t_{p, s}: s \in S\right\}} .
$$


Suppose that a parameter $r_{M} \geq r_{p, s}$ for all $p, s$ is chosen, and $r_{p, s}=r_{M}$ if and only if solver $s$ does not solve problem $p$. In order to obtain an overall assessment of the performance of the solver, they defined

$$
\rho_{s}(t)=\frac{1}{N_{p}} \operatorname{size}\left\{p \in P: r_{p, s} \leq t\right\}
$$

thus $\rho_{s}: R \rightarrow[0,1]$ was the probability for solver $s \in S$ that a performance ratio $r_{p, s}$ was within a factor $t \in R$ of the best possible ratio. tive to the number of iteration, the number of function evaluations, the number of gradient evaluations and CPU time, are evaluated using the performance profiles, respectively. Figure 1 indicates that the P-PSB method is better than P-BFGS on all aspects of performance.

Then, we compared the proposed P-PSB method with L-BFGS method based on the performance profile. The L-BFGS method constructs a matrix $H_{k}$, an approximate Hessian of $\nabla^{2} f\left(x_{k}\right)^{-1}$ by updating the basic matrix $H_{0} m$ times using the BFGS formula with previous $m$ iterations. It has been shown that the L-BFGS method converges linearly. Since the L-BFGS method does not use much information of the Hessian, it is now widely used in practice. While, it converges very slowly for ill-conditioned problems [11. In Figure 2, we give the comparison of P-PSB method and L-BFGS method relative to the number of iteration, the number of function evaluations, the number of gradient evaluations and CPU time. The top curves in the figures correspond to the

\section{L-BFGS}

At last, we compare the proposed P-PSB method with its dense version, i.e., the projected PSB method in Table 4. The preliminary numerical results show that the P-PSB method can terminate at solutions of the problems successfully. 225 While, the projected PSB fails for several problems. Moreover, it's obvious that the P-PSB method has better performance, especially in terms of CPU time. The P-PSB method can obtain solutions of problems up to $2 \times 10^{6}$ dimension , while the projected PSB method can only get solutions with dimension no more 
than 500 in a short period of time. Taking into account that the subproblem of a sparse quasi-Newton method is easier to solve than its dense version, so P-PSB method is desirable to solve large-scale problems. The results further positively supported the proposed method.

\section{Final Remarks}

We have proposed a partitioned PSB quasi-Newton method for solving parUniversities 531107040846.

\section{References}

[1] R. H. Byrd, J. Nocedal, A tool for the analysis of quasi-newton methods with application to unconstrained minimization, SIAM Journal on Numer- 
[2] R. H. Byrd, J. Nocedal, Y.-X. Yuan, Global convergence of a cass of quasinewton methods on convex problems, SIAM Journal on Numerical Analysis 24 (5) (1987) 1171-1190.

[3] D.-H. Li, M. Fukushima, On the global convergence of the bfgs method for nonconvex unconstrained optimization problems, SIAM Journal on Optimization 11 (4) (2001) 1054-1064.

[4] D.-H. Li, M. Fukushima, A modified bfgs method and its global convergence in nonconvex minimization, Journal of Computational and Applied Mathematics 129 (1) (2001) 15-35.

[5] X.-M. An, D.-H. Li, Y. Xiao, Sufficient descent directions in unconstrained optimization, Computational Optimization and Applications 48 (3) (2011) $515-532$.

[6] J. Nocedal, Updating quasi-newton matrices with limited storage, Mathematics of computation 35 (151) (1980) 773-782.

270 [7] P. L. Toint, On sparse and symmetric matrix updating subject to a linear equation, Mathematics of Computation 31 (140) (1977) 954-961.

[8] R. Fletcher, An optimal positive definite update for sparse hessian matrices, SIAM Journal on Optimization 5 (1) (1995) 192-218.

[9] Y.-H. Dai, N. Yamashita, Analysis of sparse quasi-newton updates with positive definite matrix completion, Journal of the Operations Research Society of China 2 (1) (2014) 39-56.

[10] P. Toint, On the superlinear convergence of an algorithm for solving a sparse minimization problem, SIAM Journal on Numerical Analysis 16 (6) (1979) 1036-1045.

[11] N. Yamashita, Sparse quasi-newton updates with positive definite matrix completion, Mathematical programming 115 (1) (2008) 1-30. 
[12] A. Griewank, P. L. Toint, Partitioned variable metric updates for large structured optimization problems, Numerische Mathematik 39 (1) (1982) $119-137$.

[13] A. Griewank, P. L. Toint, Local convergence analysis for partitioned quasinewton updates, Numerische Mathematik 39 (3) (1982) 429-448.

[14] P. L. Toint, Global convergence of the partitioned bfgs algorithm for convex partially separable optimization, Mathematical Programming 36 (3) (1986) 290-306.

[15] A. Griewank, The global convergence of partitioned bfgs on problems with convex decompositions and lipschitzian gradients, Mathematical programming 50 (1-3) (1991) 141-175.

[16] J. J. More, J. A. Trangenstein, On the global convergence of broydens method, Mathematics of Computation 30 (135) (1976) 523-540.

[17] E. Marwil, Convergence results for schubert's method for solving sparse nonlinear equations, SIAM Journal on Numerical Analysis 16 (4) (1979) 588-604.

[18] L. Lukšan, C. Matonoha, J. Vlcek, Modified cute problems for sparse unconstrained optimization, Techical Report 1081.

[19] N. Andrei, An unconstrained optimization test functions collection, Adv. Model. Optim 10 (1) (2008) 147-161.

[20] L. Lukšan, C. Matonoha, J. Vlcek, Sparse test problems for unconstrained optimization, Techical Report 1064.

[21] E. D. Dolan, J. J. Moré, Benchmarking optimization software with performance profiles, Mathematical programming 91 (2) (2002) 201-213. 
Table 1: Comparison on Problems 1-10

\begin{tabular}{|c|c|c|c|c|c|c|c|c|c|c|c|c|c|}
\hline \multirow[b]{2}{*}{ Pro } & \multirow[b]{2}{*}{$\mathrm{n}$} & \multicolumn{3}{|c|}{ P-PSB } & \multicolumn{4}{|c|}{ P-BFGS } & \multicolumn{4}{|c|}{ L-BFGS } & \multirow[b]{2}{*}{ time } \\
\hline & & Ite & fent & grad & time & Ite & fent & grad & time & Ite & fent & grad & \\
\hline \multirow[t]{4}{*}{1} & 1000 & 14 & 46 & 29 & 0.0000 & 33 & 86 & 68 & 0.0313 & 30 & 123 & 63 & 0.0313 \\
\hline & 10000 & 14 & 59 & 30 & 0.0938 & 33 & 86 & 68 & 0.2031 & 30 & 123 & 63 & 0.2656 \\
\hline & 100000 & 14 & 59 & 30 & 0.9844 & 33 & 86 & 68 & 1.1563 & 30 & 123 & 63 & 2.8281 \\
\hline & 1000000 & 14 & 59 & 30 & 4.8438 & 33 & 86 & 68 & 9.4063 & 30 & 123 & 63 & 25.3125 \\
\hline \multirow[t]{4}{*}{2} & 1000 & 36 & 127 & 125 & 0.1563 & 21 & 89 & 45 & 0.0313 & 20 & 97 & 62 & 0.0625 \\
\hline & 10000 & 36 & 127 & 125 & 0.8750 & 21 & 89 & 45 & 0.2031 & 20 & 97 & 62 & 0.4688 \\
\hline & 100000 & 36 & 127 & 125 & 5.9531 & 21 & 89 & 45 & 1.9531 & 20 & 97 & 62 & 3.7500 \\
\hline & 1000000 & 36 & 127 & 125 & 65.7969 & 21 & 89 & 45 & 20.0469 & 20 & 97 & 62 & 29.1563 \\
\hline \multirow[t]{4}{*}{3} & 1000 & 47 & 485 & 102 & 0.0625 & 33 & 86 & 68 & 0.0000 & 30 & 112 & 63 & 0.0313 \\
\hline & 10000 & 44 & 431 & 95 & 0.3438 & 33 & 86 & 68 & 0.1563 & 30 & 112 & 63 & 0.2500 \\
\hline & 100000 & 50 & 290 & 107 & 2.1719 & 33 & 86 & 68 & 1.2656 & 30 & 112 & 63 & 2.4688 \\
\hline & 1000000 & 49 & 285 & 104 & 21.3906 & 33 & 86 & 68 & 9.4688 & 30 & 112 & 63 & 23.0469 \\
\hline \multirow[t]{4}{*}{4} & 1000 & 2 & 9 & 5 & 0.0000 & 18 & 39 & 37 & 0.0313 & 14 & 45 & 29 & 0.0313 \\
\hline & 10000 & 2 & 9 & 5 & 0.0000 & 18 & 39 & 37 & 0.2813 & 14 & 45 & 29 & 0.1875 \\
\hline & 100000 & 2 & 9 & 5 & 0.1563 & 18 & 39 & 37 & 1.4844 & 14 & 45 & 29 & 1.6563 \\
\hline & 1000000 & 2 & 9 & 5 & 1.1719 & 18 & 39 & 37 & 13.7656 & 14 & 45 & 29 & 14.1250 \\
\hline \multirow[t]{4}{*}{5} & 1000 & 8 & 22 & 17 & 0.0313 & 11 & 41 & 23 & 0.0313 & 8 & 28 & 17 & 0.0000 \\
\hline & 10000 & 8 & 22 & 17 & 0.0938 & 11 & 41 & 23 & 0.1563 & 8 & 28 & 17 & 0.0938 \\
\hline & 100000 & 8 & 22 & 17 & 0.4219 & 11 & 41 & 23 & 1.2656 & 8 & 28 & 17 & 0.7813 \\
\hline & 1000000 & 9 & 25 & 19 & 4.5938 & 11 & 41 & 23 & 7.1094 & 9 & 58 & 35 & 16.9844 \\
\hline \multirow[t]{4}{*}{6} & 1000 & 8 & 33 & 18 & 0.0000 & 9 & 31 & 20 & 0.0313 & 7 & 33 & 15 & 0.0000 \\
\hline & 10000 & 8 & 33 & 18 & 0.0313 & 9 & 31 & 20 & 0.0625 & 7 & 33 & 15 & 0.0313 \\
\hline & 100000 & 8 & 33 & 18 & 0.2656 & 9 & 31 & 20 & 0.5625 & 7 & 33 & 15 & 0.3750 \\
\hline & 1000000 & 8 & 33 & 18 & 2.5938 & 9 & 31 & 20 & 3.0625 & 7 & 33 & 15 & 2.8750 \\
\hline \multirow[t]{4}{*}{7} & 1000 & 12 & 35 & 26 & 0.0000 & 13 & 34 & 28 & 0.0000 & 13 & 47 & 27 & 0.0000 \\
\hline & 10000 & 12 & 35 & 26 & 0.1250 & 13 & 34 & 28 & 0.0938 & 13 & 47 & 27 & 0.0938 \\
\hline & 100000 & 12 & 35 & 26 & 1.1875 & 13 & 34 & 28 & 0.7031 & 13 & 47 & 27 & 1.2969 \\
\hline & 1000000 & 12 & 35 & 26 & 6.4844 & 13 & 34 & 28 & 5.9063 & 13 & 47 & 27 & 8.7500 \\
\hline \multirow[t]{4}{*}{8} & 1000 & 15 & 79 & 34 & 0.0000 & 17 & 114 & 36 & 0.0781 & 10 & 42 & 23 & 0.0000 \\
\hline & 10000 & 12 & 63 & 27 & 0.1250 & 17 & 114 & 36 & 0.7656 & 10 & 42 & 23 & 0.0781 \\
\hline & 100000 & 21 & 116 & 49 & 1.2031 & 17 & 114 & 36 & 4.0156 & 10 & 42 & 23 & 0.8594 \\
\hline & 1000000 & 25 & 125 & 54 & 12.3125 & 17 & 114 & 36 & 37.0000 & 10 & 42 & 23 & 5.8594 \\
\hline \multirow[t]{4}{*}{9} & 1000 & 54 & 172 & 124 & 0.0313 & 66 & 243 & 142 & 0.0313 & 60 & 212 & 128 & 0.0938 \\
\hline & 10000 & 54 & 172 & 124 & 0.3750 & 66 & 243 & 142 & 0.3438 & 60 & 212 & 128 & 0.5313 \\
\hline & 100000 & 54 & 172 & 124 & 2.2969 & 66 & 243 & 142 & 1.2656 & 61 & 217 & 130 & 4.7031 \\
\hline & 1000000 & 56 & 196 & 131 & 20.3750 & 66 & 243 & 142 & 14.9219 & 61 & 217 & 130 & 58.1250 \\
\hline \multirow[t]{4}{*}{10} & 1000 & 9 & 101 & 56 & 0.0313 & 38 & 184 & 132 & 0.0313 & 21 & 241 & 175 & 0.0313 \\
\hline & 10000 & 9 & 101 & 56 & 0.1875 & 37 & 182 & 130 & 0.3438 & 18 & 235 & 172 & 0.3125 \\
\hline & 100000 & 9 & 101 & 56 & 0.8281 & 37 . & $2^{182}$ & 130 & 1.8594 & 17 & 226 & 167 & 1.8438 \\
\hline & 1000000 & 9 & 101 & 56 & 9.0469 & $37^{2}$ & 182 & 130 & 18.9063 & 17 & 228 & 167 & 21.5469 \\
\hline
\end{tabular}


Table 2: Comparison on Problems 11-20

\begin{tabular}{|c|c|c|c|c|c|c|c|c|c|c|c|c|c|}
\hline \multirow[b]{2}{*}{ Pro } & \multirow[b]{2}{*}{$\mathrm{n}$} & \multicolumn{3}{|c|}{ P-PSB } & \multirow[b]{2}{*}{ time } & \multicolumn{3}{|c|}{ P-BFGS } & \multicolumn{4}{|c|}{ L-BFGS } & \multirow[b]{2}{*}{ time } \\
\hline & & Ite & fcnt & $\operatorname{grad}$ & & Ite & fcnt & grad & time & Ite & fcnt & grad & \\
\hline \multirow[t]{4}{*}{11} & 1000 & 4 & 16 & 10 & 0.0000 & 5 & 26 & 11 & 0.0000 & 4 & 20 & 9 & 0.0000 \\
\hline & 10000 & 4 & 16 & 10 & 0.0313 & 5 & 26 & 11 & 0.0938 & 4 & 20 & 9 & 0.0313 \\
\hline & 100000 & 4 & 16 & 10 & 0.4063 & 5 & 26 & 11 & 0.5781 & 4 & 20 & 9 & 0.2500 \\
\hline & 1000000 & 4 & 16 & 10 & 2.0781 & 5 & 26 & 11 & 3.0156 & 4 & 20 & 9 & 1.6250 \\
\hline \multirow[t]{4}{*}{12} & 1000 & 2 & 7 & 5 & 0.0000 & 7 & 17 & 16 & 0.0000 & 7 & 24 & 15 & 0.0000 \\
\hline & 10000 & 2 & 7 & 5 & 0.0000 & 7 & 17 & 16 & 0.0156 & 7 & 24 & 15 & 0.0000 \\
\hline & 100000 & 2 & 7 & 5 & 0.1250 & 7 & 17 & 16 & 0.4219 & 7 & 24 & 15 & 0.2188 \\
\hline & 1000000 & 2 & 7 & 5 & 0.8281 & 7 & 17 & 16 & 1.9844 & 7 & 24 & 15 & 2.1719 \\
\hline \multirow[t]{4}{*}{13} & 1000 & 10 & 35 & 23 & 0.0000 & 11 & 68 & 29 & 0.0313 & 10 & 45 & 22 & 0.0000 \\
\hline & 10000 & 10 & 35 & 23 & 0.0938 & 11 & 68 & 29 & 0.0938 & 10 & 45 & 22 & 0.0469 \\
\hline & 100000 & 10 & 35 & 23 & 0.7344 & 11 & 68 & 29 & 1.1563 & 10 & 45 & 22 & 0.4844 \\
\hline & 1000000 & 10 & 35 & 23 & 4.0938 & 11 & 68 & 29 & 6.1563 & 10 & 45 & 22 & 4.7500 \\
\hline \multirow[t]{4}{*}{14} & 1000 & 7 & 23 & 25 & 0.0000 & 11 & 25 & 26 & 0.0313 & 7 & 30 & 24 & 0.0313 \\
\hline & 10000 & 7 & 23 & 25 & 0.0625 & 11 & 25 & 26 & 0.1250 & 7 & 30 & 24 & 0.0469 \\
\hline & 100000 & 7 & 23 & 25 & 0.8906 & 11 & 25 & 26 & 1.1875 & 7 & 30 & 24 & 0.4219 \\
\hline & 1000000 & 7 & 23 & 25 & 4.7813 & 11 & 25 & 26 & 6.5625 & 7 & 30 & 24 & 5.6250 \\
\hline \multirow[t]{4}{*}{15} & 1000 & 15 & 70 & 32 & 0.0313 & 92 & 208 & 185 & 0.1563 & 7 & 24 & 15 & 0.0000 \\
\hline & 10000 & 15 & 70 & 32 & 0.2188 & 92 & 208 & 185 & 1.3750 & 7 & 24 & 15 & 0.0313 \\
\hline & 100000 & 15 & 70 & 32 & 1.6719 & 92 & 208 & 185 & 8.7188 & 7 & 24 & 15 & 0.3438 \\
\hline & 1000000 & 15 & 70 & 32 & 16.6875 & 92 & 208 & 185 & 86.0313 & 7 & 24 & 15 & 2.5781 \\
\hline \multirow[t]{4}{*}{16} & 1000 & 8 & 23 & 18 & 0.0000 & 12 & 52 & 27 & 0.0313 & 40 & 646 & 115 & 0.2188 \\
\hline & 10000 & 8 & 23 & 18 & 0.1563 & 12 & 52 & 27 & 0.2188 & 59 & 1253 & 137 & 1.8906 \\
\hline & 100000 & 8 & 23 & 18 & 0.7969 & 12 & 52 & 27 & 1.3125 & 59 & 1253 & 137 & 15.4688 \\
\hline & 1000000 & 8 & 23 & 18 & 7.6250 & 12 & 52 & 27 & 12.8594 & 59 & 1254 & 138 & 158.9844 \\
\hline \multirow[t]{4}{*}{17} & 1000 & 39 & 275 & 85 & 0.0313 & 75 & 207 & 162 & 0.0625 & 71 & 269 & 155 & 0.0625 \\
\hline & 10000 & 76 & 789 & 159 & 0.6094 & 75 & 207 & 162 & 0.3750 & 71 & 269 & 156 & 0.3906 \\
\hline & 100000 & 60 & 604 & 127 & 3.0781 & 75 & 207 & 162 & 2.7813 & 70 & 264 & 152 & 2.6563 \\
\hline & 1000000 & 62 & 648 & 130 & 28.8906 & 75 & 207 & 162 & 23.1719 & 71 & 270 & 156 & 29.7031 \\
\hline \multirow[t]{4}{*}{18} & 1000 & 21 & 71 & 55 & 0.0313 & 71 & 522 & 146 & 0.0625 & 22 & 96 & 56 & 0.0000 \\
\hline & 10000 & 21 & 71 & 55 & 0.0938 & 67 & 527 & 139 & 0.5313 & 22 & 96 & 56 & 0.1250 \\
\hline & 100000 & 21 & 71 & 55 & 0.8281 & 67 & 527 & 138 & 3.3594 & 22 & 96 & 56 & 0.8125 \\
\hline & 1000000 & 21 & 71 & 55 & 7.1094 & 66 & 520 & 136 & 30.6094 & 22 & 96 & 56 & 9.3750 \\
\hline \multirow[t]{4}{*}{19} & 1000 & 49 & 186 & 100 & 1.9063 & 41 & 105 & 85 & 0.8750 & 41 & 144 & 83 & 0.0938 \\
\hline & 10000 & 38 & 164 & 78 & 8.8594 & 41 & 105 & 85 & 5.7813 & 42 & 147 & 85 & 0.7188 \\
\hline & 100000 & 37 & 162 & 76 & 74.0938 & 45 & 113 & 93 & 60.9531 & 42 & 147 & 85 & 5.1094 \\
\hline & 1000000 & 36 & 160 & 74 & 670.6406 & 45 & 113 & 93 & 575.7188 & 54 & 196 & 111 & 72.8125 \\
\hline \multirow[t]{4}{*}{20} & 1000 & 36 & 110 & 75 & 0.6875 & 49 & 209 & 118 & 1.2500 & 27 & 101 & 55 & 0.0313 \\
\hline & 10000 & 36 & 110 & 75 & 4.6875 & 49 & 209 & 118 & 9.1563 & 27 & 101 & 55 & 0.1875 \\
\hline & 100000 & 36 & 110 & 75 & 19.3961 & 49 & 209 & 118 & 71.4526 & 27 & 101 & 55 & 2.0625 \\
\hline & 1000000 & 36 & 110 & 75 & 26.5590 & 49 & 209 & 118 & 91.7188 & 27 & 101 & 55 & 20.1875 \\
\hline
\end{tabular}


Table 3: Comparison on Problems 21-30

\begin{tabular}{|c|c|c|c|c|c|c|c|c|c|c|c|c|c|}
\hline \multirow[b]{2}{*}{ Pro } & \multirow[b]{2}{*}{$\mathrm{n}$} & \multicolumn{4}{|c|}{ P-PSB } & \multicolumn{4}{|c|}{ P-BFGS } & \multicolumn{4}{|c|}{ L-BFGS } \\
\hline & & Ite & fent & grad & time & Ite & fcnt & grad & time & Ite & fcnt & grad & time \\
\hline \multirow[t]{4}{*}{21} & 1000 & 76 & 1440 & 205 & 0.4063 & 129 & 2317 & 143 & 0.2500 & 16 & 56 & 34 & 0.0313 \\
\hline & 10000 & 93 & 1443 & 197 & 2.1250 & 121 & 2102 & 187 & 1.5781 & 14 & 49 & 29 & 0.1875 \\
\hline & 100000 & 77 & 1322 & 160 & 12.0469 & 97 & 1715 & 151 & 9.7031 & 12 & 43 & 25 & 0.5313 \\
\hline & 1000000 & 87 & 1192 & 113 & 82.6094 & 71 & 1318 & 178 & 93.2344 & 11 & 40 & 23 & 6.6875 \\
\hline \multirow[t]{4}{*}{22} & 1000 & 10 & 26 & 24 & 0.0625 & 10 & 27 & 23 & 0.0000 & 8 & 35 & 19 & 0.0313 \\
\hline & 10000 & 22 & 51 & 27 & 0.3125 & 12 & 30 & 27 & 0.0938 & 7 & 32 & 17 & 0.0625 \\
\hline & 100000 & 6 & 19 & 15 & 0.9688 & 11 & 28 & 25 & 0.7813 & 7 & 30 & 17 & 0.3906 \\
\hline & 1000000 & 4 & 14 & 11 & 3.6719 & 10 & 26 & 23 & 5.8438 & 15 & 54 & 33 & 9.6719 \\
\hline \multirow[t]{4}{*}{23} & 1000 & 6 & 12 & 13 & 0.0000 & 7 & 14 & 15 & 0.0000 & 7 & 21 & 15 & 0.0000 \\
\hline & 10000 & 6 & 12 & 13 & 0.0313 & 7 & 14 & 15 & 0.0313 & 7 & 21 & 15 & 0.0313 \\
\hline & 100000 & 6 & 12 & 13 & 0.3438 & 7 & 14 & 15 & 0.3281 & 7 & 21 & 15 & 0.2813 \\
\hline & 1000000 & 6 & 12 & 13 & 1.9063 & 8 & 16 & 17 & 1.8750 & 8 & 24 & 17 & 3.3438 \\
\hline \multirow[t]{4}{*}{24} & 1000 & 5 & 11 & 11 & 0.0313 & 5 & 11 & 11 & 0.0000 & 5 & 16 & 11 & 0.0000 \\
\hline & 10000 & 5 & 11 & 11 & 0.0313 & 5 & 11 & 11 & 0.0313 & 5 & 16 & 11 & 0.0313 \\
\hline & 100000 & 5 & 11 & 11 & 0.3438 & 5 & 11 & 11 & 0.3125 & 5 & 16 & 11 & 0.2500 \\
\hline & 1000000 & 5 & 11 & 11 & 1.8438 & 5 & 11 & 11 & 1.6094 & 5 & 16 & 11 & 2.0938 \\
\hline \multirow[t]{4}{*}{25} & 1000 & 4 & 8 & 9 & 0.0000 & 5 & 10 & 11 & 0.0313 & 5 & 15 & 11 & 0.0313 \\
\hline & 10000 & 4 & 8 & 9 & 0.0313 & 5 & 10 & 11 & 0.0625 & 5 & 15 & 11 & 0.0625 \\
\hline & 100000 & 4 & 8 & 9 & 0.4375 & 5 & 10 & 11 & 0.4844 & 5 & 15 & 11 & 0.5313 \\
\hline & 1000000 & 4 & 8 & 9 & 2.0000 & 5 & 10 & 11 & 2.4375 & 5 & 15 & 11 & 3.4844 \\
\hline \multirow[t]{4}{*}{26} & 1000 & 6 & 13 & 13 & 0.0000 & 6 & 13 & 13 & 0.0313 & 6 & 19 & 13 & 0.0000 \\
\hline & 10000 & 6 & 13 & 13 & 0.0313 & 6 & 13 & 13 & 0.0313 & 6 & 19 & 13 & 0.0313 \\
\hline & 100000 & 6 & 13 & 13 & 0.3438 & 7 & 15 & 15 & 0.3125 & 7 & 22 & 15 & 0.2188 \\
\hline & 1000000 & 6 & 13 & 13 & 1.7656 & 7 & 15 & 15 & 1.9375 & 7 & 22 & 15 & 3.1406 \\
\hline \multirow[t]{4}{*}{27} & 1000 & 13 & 26 & 27 & 0.0000 & 13 & 26 & 27 & 0.0313 & 243 & 3593 & 395 & 0.7188 \\
\hline & 10000 & 13 & 26 & 27 & 0.0938 & 13 & 26 & 27 & 0.0938 & 90 & 1163 & 156 & 1.4844 \\
\hline & 100000 & 13 & 26 & 27 & 0.5781 & 13 & 26 & 27 & 0.6250 & 59 & 853 & 100 & 8.1875 \\
\hline & 1000000 & 13 & 26 & 27 & 4.7813 & 13 & 26 & 27 & 5.4531 & 36 & 487 & 66 & 50.6875 \\
\hline \multirow[t]{4}{*}{28} & 1000 & 2 & 10 & 5 & 0.0000 & 3 & 18 & 7 & 0.0000 & 9 & 33 & 19 & 0.0313 \\
\hline & 10000 & 2 & 10 & 5 & 0.0313 & 3 & 18 & 7 & 0.0000 & 9 & 33 & 19 & 0.0625 \\
\hline & 100000 & 2 & 10 & 5 & 0.0781 & 3 & 18 & 7 & 0.1563 & 10 & 36 & 21 & 0.8906 \\
\hline & 1000000 & 2 & 10 & 5 & 0.3906 & 3 & 18 & 7 & 0.7656 & 10 & 36 & 21 & 7.2188 \\
\hline \multirow[t]{4}{*}{29} & 1000 & 15 & 40 & 31 & 0.0000 & 32 & 67 & 65 & 0.0625 & 18 & 58 & 38 & 0.0313 \\
\hline & 10000 & 13 & 35 & 27 & 0.1563 & 26 & 55 & 53 & 0.2344 & 13 & 41 & 27 & 0.0625 \\
\hline & 100000 & 27 & 39 & 55 & 1.8281 & 20 & 43 & 41 & 1.1875 & 8 & 26 & 17 & 0.3594 \\
\hline & 1000000 & 28 & 52 & 68 & 19.7031 & 14 & 31 & 29 & 8.1563 & 6 & 20 & 13 & 2.4375 \\
\hline \multirow[t]{4}{*}{30} & 1000 & 55 & 215 & 112 & 0.0625 & 18 & 44 & 38 & 0.0313 & 27 & 87 & 57 & 0.0313 \\
\hline & 10000 & 41 & 303 & 84 & 0.5469 & 18 & 44 & 38 & 0.1875 & 31 & 101 & 63 & 0.3125 \\
\hline & 100000 & 21 & 132 & 43 & 1.3906 & 18 & 44 & 38 & 1.0156 & 33 & 112 & 70 & 2.7344 \\
\hline & 1000000 & 10 & 50 & 21 & 3.8125 & 18 & 44 & 38 & 10.0781 & 42 & 147 & 86 & 38.1719 \\
\hline
\end{tabular}


Table 4: Numerical Results of PSB Method

\begin{tabular}{|c|c|c|c|c|c|c|c|c|c|c|c|c|c|c|c|c|}
\hline \multirow[b]{2}{*}{$\mathrm{n}$} & \multicolumn{4}{|c|}{ Pro 4} & \multicolumn{4}{|c|}{ Pro 5} & \multicolumn{4}{|c|}{ Pro 6} & \multicolumn{4}{|c|}{ Pro 7} \\
\hline & Ite & fent & grad & Time & Ite & fent & grad & Time & Ite & fent & grad & Time & Ite & fent & grad & Time \\
\hline 10 & 2 & 9 & 5 & 0.0000 & 15 & 55 & 31 & 0.0156 & 10 & 45 & 23 & 0.0000 & 18 & 52 & 38 & 0.0313 \\
\hline 50 & 2 & 9 & 5 & 0.0313 & 19 & 75 & 39 & 0.0625 & 65 & 446 & 133 & 0.2188 & 12 & 35 & 26 & 0.0313 \\
\hline 100 & 2 & 9 & 5 & 0.0313 & 19 & 75 & 39 & 0.3281 & 68 & 471 & 141 & 1.0625 & 12 & 35 & 26 & 0.1875 \\
\hline \multirow[t]{2}{*}{500} & 2 & 9 & 5 & 2.3281 & 15 & 53 & 31 & 17.5313 & 119 & 562 & 256 & 17.5623 & 12 & 35 & 26 & 14.1406 \\
\hline & \multicolumn{4}{|c|}{ Pro 8} & \multicolumn{4}{|c|}{ Pro 10} & \multicolumn{4}{|c|}{ Pro 12} & \multicolumn{4}{|c|}{ Pro 13} \\
\hline $\mathrm{n}$ & Ite & fent & grad & Time & Ite & fent & grad & Time & Ite & fent & grad & Time & Ite & fent & grad & Time \\
\hline 10 & 38 & 172 & 79 & 0.0313 & 9 & 101 & 56 & 0.0000 & 5 & 26 & 11 & 0.0000 & 2 & 7 & 5 & 0.0000 \\
\hline 50 & 40 & 219 & 82 & 0.1563 & 11 & 111 & 60 & 0.0625 & 5 & 26 & 11 & 0.0313 & 2 & 7 & 5 & 0.0000 \\
\hline 100 & 38 & 164 & 79 & 0.6094 & 11 & 111 & 60 & 0.1875 & 5 & 26 & 11 & 0.0625 & 2 & 7 & 5 & 0.0625 \\
\hline \multirow[t]{2}{*}{500} & 64 & 312 & 131 & 75.0625 & 13 & 128 & 64 & 16.0313 & 5 & 26 & 11 & 5.9531 & 2 & 7 & 5 & 2.4531 \\
\hline & \multicolumn{4}{|c|}{ Pro 15} & \multicolumn{4}{|c|}{ Pro 16} & \multicolumn{4}{|c|}{ Pro 21} & \multicolumn{4}{|c|}{ Pro 22} \\
\hline $\mathrm{n}$ & Ite & fent & grad & Time & Ite & fent & grad & Time & Ite & fent & grad & Time & Ite & fent & grad & Time \\
\hline 10 & 11 & 25 & 26 & 0.0313 & 9 & 30 & 21 & 0.0000 & 32 & 169 & 64 & 0.0313 & 10 & 26 & 20 & 0.0000 \\
\hline 50 & 11 & 25 & 26 & 0.0313 & 8 & 28 & 19 & 0.0313 & 127 & 889 & 260 & 0.5313 & 22 & 39 & 47 & 0.8125 \\
\hline 100 & 11 & 25 & 26 & 0.2500 & 8 & 28 & 19 & 0.1094 & 181 & 1428 & 366 & 3.2188 & 18 & 53 & 36 & 1.3750 \\
\hline \multirow[t]{2}{*}{500} & 11 & 25 & 26 & 14.1719 & 8 & 28 & 19 & 10.2656 & 226 & 1986 & 530 & 16.3988 & 14 & 64 & 30 & 17.2031 \\
\hline & \multicolumn{4}{|c|}{ Pro 23} & \multicolumn{4}{|c|}{ Pro 24} & \multicolumn{4}{|c|}{ Pro 25} & \multicolumn{4}{|c|}{ Pro 26} \\
\hline $\mathrm{n}$ & Ite & fent & grad & Time & Ite & fent & grad & Time & Ite & fent & grad & Time & Ite & fent & grad & Time \\
\hline 10 & 25 & 161 & 50 & 0.0000 & 26 & 106 & 52 & 0.0313 & 7 & 41 & 14 & 0.0000 & 5 & 11 & 11 & 0.0000 \\
\hline 50 & 74 & 732 & 150 & 0.2969 & 35 & 142 & 70 & 0.1250 & 9 & 58 & 18 & 0.0313 & 5 & 11 & 11 & 0.0313 \\
\hline 100 & 129 & 1384 & 266 & 2.1094 & 36 & 151 & 72 & 0.6094 & 9 & 55 & 18 & 0.1563 & 5 & 11 & 11 & 0.0938 \\
\hline \multirow[t]{2}{*}{500} & 344 & 3541 & 697 & 408.5938 & 39 & 185 & 80 & 46.4063 & 9 & 61 & 18 & 11.3906 & 5 & 11 & 11 & 6.0156 \\
\hline & \multicolumn{4}{|c|}{ Pro 27} & \multicolumn{4}{|c|}{ Pro 28} & \multicolumn{4}{|c|}{ Pro 29} & \multicolumn{4}{|c|}{ Pro 30} \\
\hline $\mathrm{n}$ & Ite & fent & grad & Time & Ite & fent & grad & Time & Ite & fent & grad & Time & Ite & fent & grad & Time \\
\hline 10 & 4 & 14 & 9 & 0.0313 & 6 & 40 & 12 & 0.0313 & 42 & 111 & 84 & 0.2813 & 6 & 51 & 13 & 0.0938 \\
\hline 50 & 4 & 14 & 9 & 0.0000 & 6 & 40 & 12 & 0.0000 & 179 & 358 & 359 & 0.7813 & 6 & 51 & 13 & 0.6094 \\
\hline 100 & 4 & 14 & 9 & 0.0781 & 6 & 40 & 12 & 0.1719 & 441 & 882 & 883 & 7.8438 & 6 & 51 & 13 & 2.7031 \\
\hline 500 & 4 & 14 & 9 & 4.7813 & 8 & 71 & 15 & 10.0781 & 563 & 1026 & 1183 & 15.3620 & 6 & 51 & 12 & 7.1094 \\
\hline
\end{tabular}


(a)

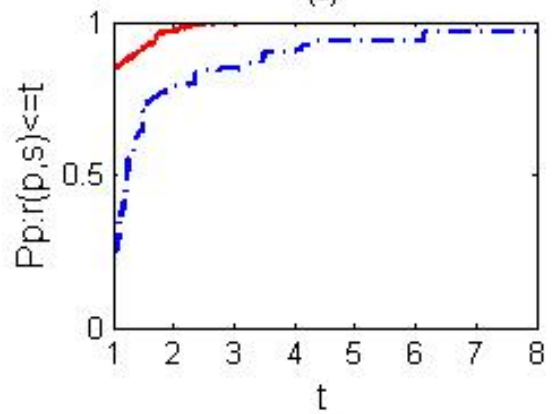

(c)

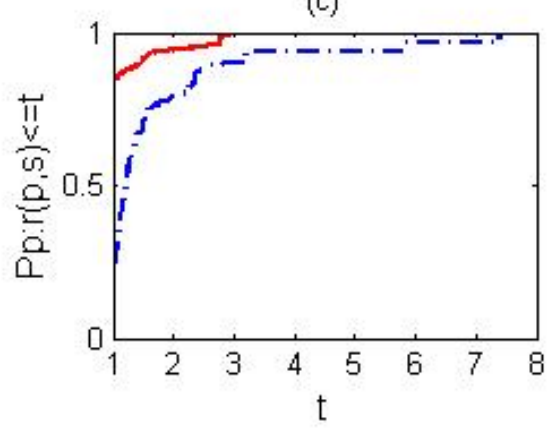

(b)

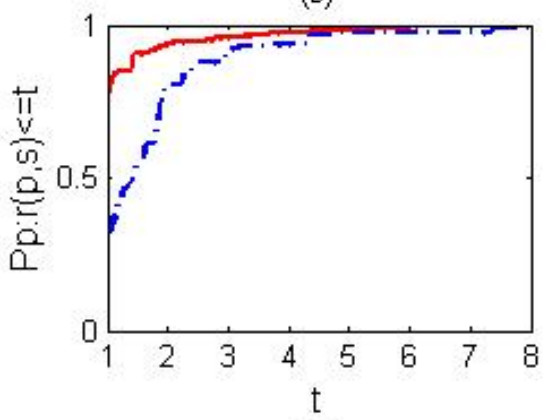

(d)

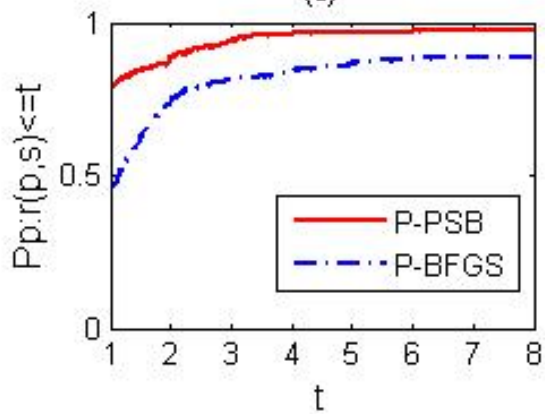

Figure 1: Performance profiles for P-PSB and P-BFGS

(a)Results comparison on the number of iteration, (b) Results comparison on the number of function evaluations, (c) Results comparison on the number of gradient evaluation, (d) Results comparison on CPU time. 
(a)

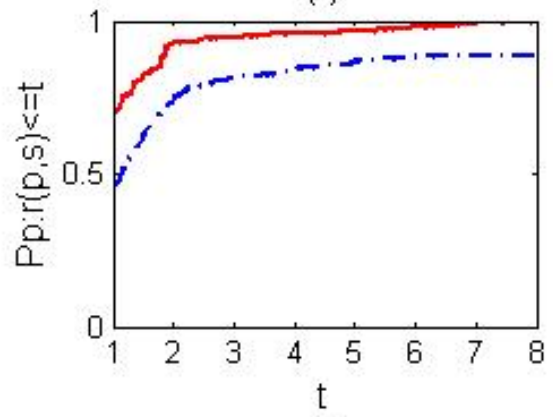

(c)



(b)



(d)



Figure 2: Performance profiles for P-PSB and L-BFGS

(a)Results comparison on the number of iteration, (b) Results comparison on the number of function evaluations, (c) Results comparison on the number of gradient evaluation, (d) Results comparison on CPU time. 